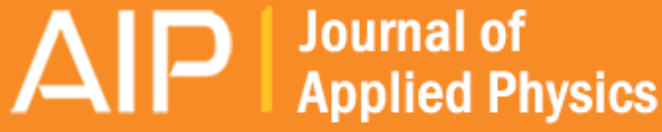

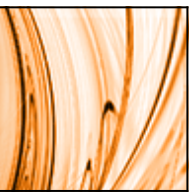

\section{ImmersedFoil Method for Measuring Shock Wave Profiles in Solids}

Thomas J. Ahrens and M. H. Ruderman

Citation: Journal of Applied Physics 37, 4758 (1966); doi: 10.1063/1.1708133

View online: http://dx.doi.org/10.1063/1.1708133

View Table of Contents: http://scitation.aip.org/content/aip/journal/jap/37/13?ver=pdfcov

Published by the AIP Publishing

\section{Articles you may be interested in}

Shock induced melting in aluminum: Wave profile measurements

AIP Conf. Proc. 505, 97 (2000); 10.1063/1.1303430

Method for the measurement of temperature in shock compression of solids

Rev. Sci. Instrum. 51, 750 (1980); 10.1063/1.1136296

Shock wave measurements on solid hydrogen and argon

J. Chem. Phys. 59, 3517 (1973); 10.1063/1.1680513

Steady shock profile in solids

J. Appl. Phys. 44, 4013 (1973); 10.1063/1.1662888

Instrument to Measure Density Profiles behind Shock Waves

Rev. Sci. Instrum. 32, 292 (1961); 10.1063/1.1717347

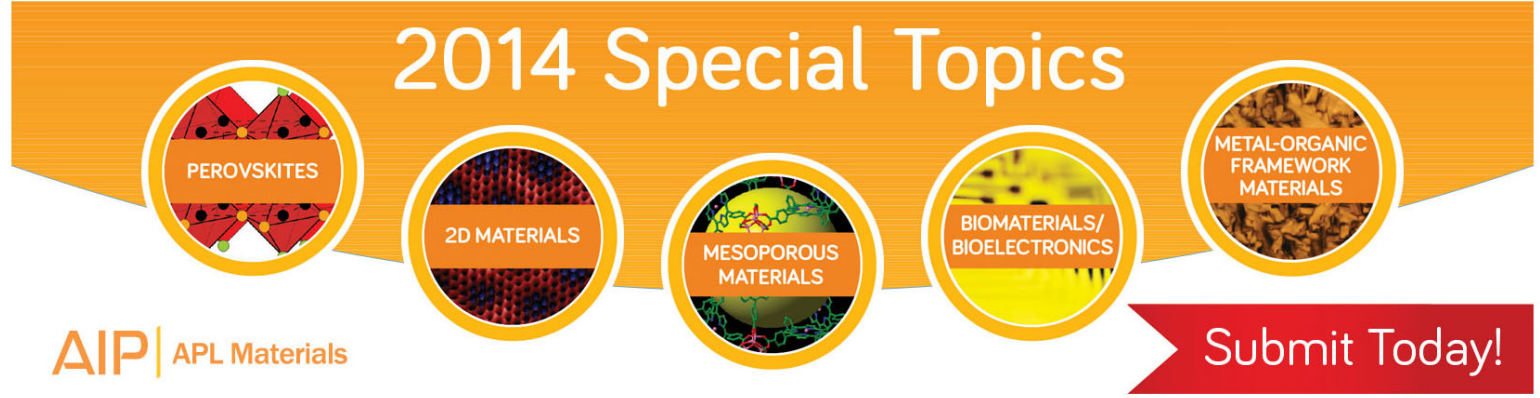


Substituting in $\mathrm{Eq}$. (41) and comparing with Eq. (40) gives

a

$$
\left[\begin{array}{lll}
A_{11}(B) & A_{12}(B) & A_{13}(B) \\
A_{21}(B) & A_{22}(B) & A_{23}(B) \\
A_{31}(B) & A_{32}(B) & A_{33}(B)
\end{array}\right]=\left[\begin{array}{rrr}
\tilde{A}_{11}(-B) & \widetilde{A}_{21}(-B) & -\tilde{A}_{31}(-B) \\
\widetilde{A}_{12}(-B) & \widetilde{A}_{22}(-B) & -\tilde{A}_{32}(-B) \\
-\widetilde{A}_{13}(-B) & -\widetilde{A}_{23}(-B) & \widetilde{A}_{33}(-B)
\end{array}\right],
$$

$\mathrm{b}$

$$
\begin{aligned}
& {\left[\begin{array}{lll}
A_{11}(B) & A_{12}(B) & A_{13}(B) \\
A_{21}(B) & A_{22}(B) & A_{23}(B) \\
A_{31}(B) & A_{32}(B) & A_{33}(B)
\end{array}\right]=\left[\begin{array}{rrr}
\tilde{A}_{11}(-B) & \widetilde{A}_{21}(-B) & \tilde{A}_{31}(-B) \\
\widetilde{A}_{12}(-B) & \widetilde{A}_{22}(-B) & \widetilde{A}_{32}(-B) \\
\widetilde{A}_{13}(-B) & \widetilde{A}_{23}(-B) & \widetilde{A}_{33}(-B)
\end{array}\right],} \\
& \mathrm{C} \\
& {\left[\begin{array}{lll}
A_{11}(B) & A_{12}(B) & A_{13}(B) \\
A_{21}(B) & A_{22}(B) & A_{23}(B) \\
A_{31}(B) & A_{32}(B) & A_{33}(B)
\end{array}\right]=\left[\begin{array}{rrr}
\widetilde{A}_{11}(-B) & -\widetilde{A}_{21}(-B) & -\widetilde{A}_{31}(-B) \\
-\tilde{A}_{12}(-B) & \widetilde{A}_{22}(-B) & \widetilde{A}_{32}(-B) \\
-\widetilde{A}_{13}(-B) & \widetilde{A}_{23}(-B) & \widetilde{A}_{33}(-B)
\end{array}\right] .}
\end{aligned}
$$

Equations (51), (52), and (53) are the transformed Onsager relations. It is evident from these equations that the arrangement of the forces in the original phenomenological equations is a relevant factor.

\title{
Immersed-Foil Method for Measuring Shock Wave Profiles in Solids
}

\author{
Thomas J. Ahrens and M. H. Ruderman \\ Poulter Laboratories, Stanford Research Insiinte, Menlo Park, California
}

(Received 4 May 1966)

\begin{abstract}
A new technique is described for measuring, essentially directly, a complex shock front profile that is transmitted into a transparent liquid from a solid material. The image of a grid light source, reflected from a Mylar foil immersed within the liquid, is recorded with a streak camera. Index of refraction data for liquids in the high-pressure shocked state (required for calculating particle velocities from image displacements) are reported for glycerol, ethanol, water, and hexane to $220,135,58$, and $41 \mathrm{kbar}$, respectively. The increase of index of refraction with density for water agrees with that reported by Zel'dovich, Sinitsyn, and Kormer, but is considerably less (as are also the results for the other liquids) than that predicted by the Lorentz-Lorenz formula. Hugoniot data obtained concurrently with the refractive index data agree closely with those of Rice and Walsh. Applications of the immersed-foil method are discussed for: (a) determining the release adiabats from shock states produced by a double shock front in a solid; (b) the study of shock wave attenuation in solids; and (3) measurement of the adiabatic sound velocity of liquids in the highpressure shocked state.
\end{abstract}

\section{INTRODUCTION}

$T$ HE immersed-foil technique was developed to determine multiple (usually two) shock states induced by a succession of shock fronts transmitted in to liquids from solids. ${ }^{1}$ Because of the continuity of pressure and particle velocity at the solid-liquid interface, the shock pressure and particle velocity associated with each shock front transmitted into the liquid will be equal to the pressure and particle velocity associated with each wave which is reflected back into the solid. In the usual case with nonporous solids in contact with liquids, these reflections are rarefactions and cause the shock pressure within the solid to be reduced adiabatically. Hence the value of the pressure and particle

\footnotetext{
${ }^{1}$ Multiple shock fronts may form in solids in certain shock pressure ranges as a result of dymamic yielding and polymorphism.
}

velocity associated with the shock transmitted into the liquid corresponds to a point on the release adiabat of the solid centered at the shock state. By determining the shock and particle velocity resulting from a succession of shock fronts transmitted into the liquid from a solid, points on the release adiabat associated with each of the shock fronts of the solid are obtained. Experiments are usually performed in plane-wave, onedimensional geometry using liquids of varying shock impedance. In this way a series of points on each of the release adiabats from each intermediate shock state of the solid is obtained.

The immersed foil method permits measurement of shock and particle velocity wavefront profiles within the liquid within a fraction of a millimeter of the solidliquid interface on a submicrosecond time scale. This capability is desirable for release adiabat measurements 
because, when multiple shock fronts are transmitted into normal liquids, the final (highest-pressure) shock front overtakes-and hence in terms of recordability obliterates-the preceding shock fronts. Measuring liquid shock states close to the solid liquid interface is also necessary to minimize attenuation effects which are present when the final shock state is induced in the solid by an in-contact explosive rather than by fyer plate impact. For the in-contact systems, the shock pressure decreases slightly with time behind the final shock front; thus some attenuation of the peak shock pressure with propagation path length is expected.

\section{EXPERIMENTAL TECHNIQUE}

The essential elements of the optical (streak camera) system used to measure the shock pressure profiles transmitted by solid specimens into various liquids are shown in Fig. 1. The method relies upon retaining suffcient transparency through various liquids, as these are subjected to shock pressures, to permit transmission of a specular optical image. The feasibility of the method was suggested by the work of Walsh and Rice, who reported the transparency of benzene, water, and ethanol up to $\sim 100 \mathrm{kbar} .{ }^{2}$

The optical lever technique used here was derived from the method devised by Fowles for observing the interaction of an oblique shock with a polished metal free surface. ${ }^{3}$ In the present method, the reflection of a grid light source, placed at a distance $h$ from a plate glass window, is obtained from a $\sim 0.01$-mm-thick aluminized Mylar foil which is immersed in the liquid behind the window. These three elements are placed at an angle $\alpha$ to the liquid-solid interface (which is in the expected plane of the shock). A liquid container, or cell, is constructed of Lucite tubing and plate glass

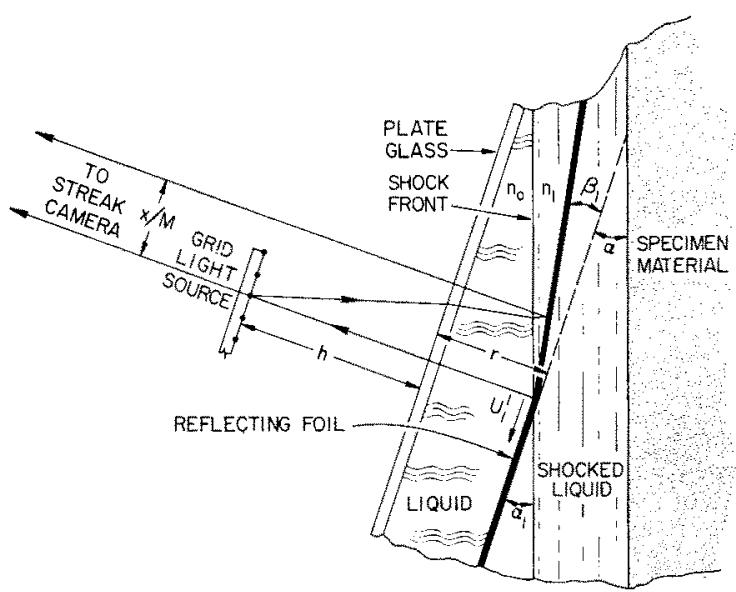

Frg. 1. Elements, in plane perpendicular to shock front, of immersed foil technique. Reflecting foll immersed, $\alpha_{1}$ to shock, in liquid of optical index $m_{0}$. After interaction with shock, foil rotated by $\beta_{1}$.

2 J. M. Walsh and M. H. Rice, J. Chem. Phys. 26, 815 (1957).

${ }^{3}$ G. R. Fowles, J. Appl. Phys. 32, 4175 (1961).

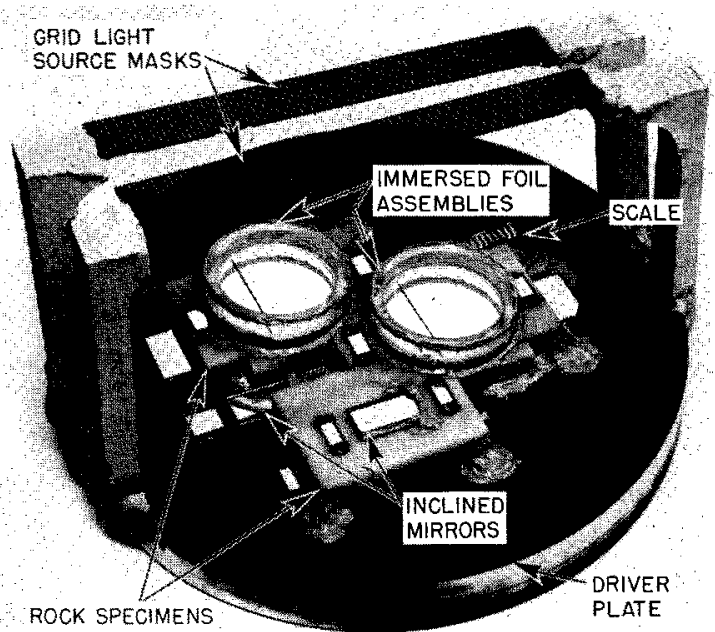

Fig. 2. Release adiabat cxperimental assembly. The explosive, which is placed below the experimental assembly, transmits a plane shock into the driver plate. Grid light source masks are illuminated from above by explosive argon light source. Markings on scale are $\frac{1}{10}$ in. apart.

cemented together with epoxy. ${ }^{4}$ The grid masks (Fig. 2) are illuminated by the ustal argon explosive light source. ${ }^{5}$

The assembly is viewed by a rotating-mirror streak camera from the direction perpendicular to the initial plane of the Mylar foil. The velocity of one or more shock fronts or of the heads of rarefaction fans in the liquid is determined from the speed with which these intersect the inclined foil. The particle velocity associated with one or more shock or rarefaction waves is determined from the displacements of the grid images as reflected in the inclined foil ; the grid image displacements, in turn, may be related to the angle each of the shock or rarefaction waves rotates the inclined foil. The foil is assumed to be sufficiently thin that it attains the particle velocity of the liquid in a time interval too short to resolve with the present streak camera $(<0.01 \mu \mathrm{sec})$. Since the grid-image displacement is also a function of the index of refraction of both the unshocked and shocked liquid, the index of the latter must be determined in separate experiments; these are described in a later section.

The streak record shown in Fig. 3 was obtained from a gylcerol liquid cell placed on top of a specimen of Arkansas novaculite (polycrystalline quartz rock) into which a 145 -kbar shock wave had been driven. A thin sheet of Mylar was placed between the liquid and the rock prevent the latter from absorbing the glycerol. The 25-kbar shock transmitted into the glycerol resulted from the 72 -kbar elastic shock in the rock. The $59-\mathrm{kbar}$ second shock in the glycerol resulted from the

${ }^{4}$ Epon 911-F, Shell Chemical Company.

${ }^{5} \mathrm{Z}$. Pressman, Proceedings of the 5 th International Conference of High-Speed Pholography, J. S. Courtney-Pratt, Ed. (Soc. of Motion Picture and Television Engineers, 1962). 


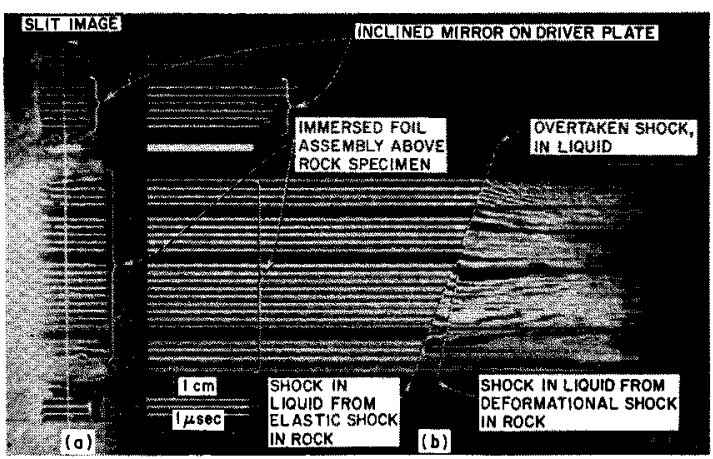

FIG. 3. Streak camera record, release adiabat experiment. (a) Photo of shot assembly taken through streak camera (still), (b) resulting streak record, shot 10635 .

145-kbar final (deformational) shock state in the rock. These are indicated on the streak record. The single forward-going shock is observed after the second (59kbar) shock in the liquid overtakes the first (25-kbar) shock in the liquid. The experimental assembly corresponding to the streak record of Fig. 3 is a simplified version of the usual more complete assembly of Fig. $2,{ }^{6}$ in which shock front profiles are measured in two (usually different) liquids; Hugoniot data are obtained using the usual inclined-mirror recording technique. ${ }^{7}$

\section{ANALYSIS OF TWO SHOCK FRONTS INCIDENT ON LIQUID CELL}

The intersection velocity along the foil of the first (or a single) shock front $U_{1}{ }^{\prime}$ is related to the shock velocity $U_{1}$ by $^{8}$

$$
U_{1}=U_{1}{ }^{\prime} \sin \alpha_{1},
$$

where $\alpha_{1}$ corresponds to the actual intersection angle of the shock with the foil. The velocity $U_{1}{ }^{\prime}$ is related

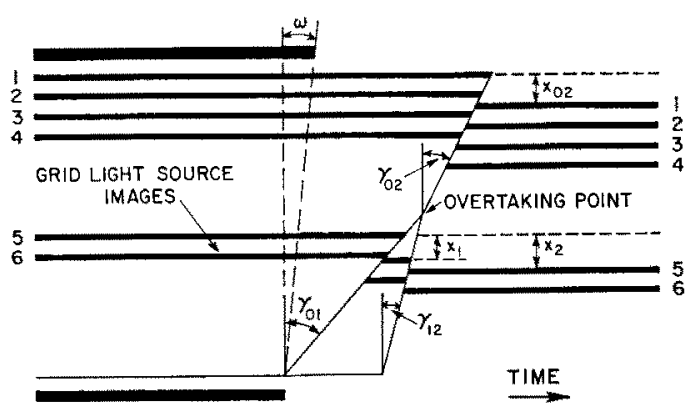

FIG. 4. Diagram of typical streak camera record, showing film angles and displacements resulting from two shock fronts propagating within immersed foil assembly.

${ }^{6}$ T. J. Ahrens and M. H. Ruderman, Stanford Research Institute, Project 4816, Contract DA-49-146-XZ-277, Interim Final Report, 30, April 1965.

7T. J. Ahrens and V. G. Gregson, Jr., J. Geophys. Res. 69, 4833 (1964).

B For quantities not defined in text, see Figs. 1 and 4 . to the film angle $\gamma_{01}$ (Fig. 4) by

$$
U_{1}^{\prime}=W \cos \alpha / M \tan \gamma_{01},
$$

where $W$ is the streak camera writing rate and $M$ is the ratio of a length on the film to the corresponding length on the shot assembly as measured in the direction parallel to the liquid-specimen interface, e.g. using the scale in Fig. 2. In general the plane of the shock is not parallel to the specimen-liquid interface but lies at a small angle $\Omega_{1}$ to it; this obliquity is recorded on the film as the angle $\omega$. It is convenient to take $\omega$ as positive when its sense of rotation is opposite to that of $\gamma_{01}$. Combining Eqs. (1) and (2) and taking into account the shock tilt as measured on the film, gives ${ }^{6}$

$$
U_{1}=\frac{W \cos \Omega_{1} \cos \alpha \sin \alpha}{M\left(\tan \gamma_{01}-\cos ^{2} \alpha \tan \omega\right)},
$$

where $\Omega_{1}$ is related to $\omega$ by

$$
\Omega_{1}=\sin ^{-1}\left[(M / W) U_{1} \tan \omega\right] .
$$

The actual intersection angle of the shock with the inclined foil is given by

$$
\alpha_{1}=\alpha+\Omega_{1} .
$$

In the usual case $\Omega_{1}$ is sufficiently small that $\cos \Omega_{1} \cong 1$. Thus $U_{1}$ may be obtained directly from $\mathrm{Eq}$. (3). In many cases $\alpha$ is sufficiently small that Eqs. (3) and (4) may be further simplified using the usual small angle approximations.

The particle velocity imparted to the liquid by a single shock is related to the foil turning angle, $\beta_{1}$, by

$$
u_{1}=U_{1}\left[1-\tan \left(\alpha_{1}-\beta_{1}\right) / \tan \alpha_{1}\right] .
$$

The turning angle of the foil is related to the image displacement $x_{1}$, as recorded on the film by

$$
x_{1} \cong M\left[\left(h n_{0} \sin g\right)\left(1-n_{0}^{2} \sin ^{2} g\right)^{-\frac{1}{2}}+r \tan g\right] / \cos \alpha,
$$

where

$$
g=\alpha_{1}+\sin ^{-1}\left\{\frac{n_{1}}{n_{0}} \sin \left[2 \beta_{1}-2 \alpha_{1}+\sin ^{-1}\left(\frac{n_{0}}{n_{1}} \sin \alpha_{1}\right)\right]\right\} .
$$

In Eq. (7) the approximation is made that the index of refraction of the glass cover of the cell is equal to the index of refraction of the unshocked liquid.

In cases where $\alpha$ is greater than $\sim 8^{\circ}$, small angle approximations for solution of Eqs. (7) and (8) may not be used. In these cases $\mathrm{Eq}$. (7) is solved numerically for the parameter $g$. Then $\mathrm{Eq}$. (8) is solved for $\beta_{1}$, using a value of $n_{1}$ which is required to be consistent with the parameters of the calculated shocked state. In order to satisfy this condition, the dependence of $n_{1}$ on a parameter(s) of the high-pressure shocked state of the liquid is required.

When $\alpha$ is a small angle, small angle approximations 
may be applied to Eqs. (6), (7), and (8); these may then be reduced and combined to yield

$$
\beta_{1} \cong \alpha_{1}+\left(n_{0} / n_{1}\right)\left\{\left[x_{1} / 2 M\left(r+n_{0} h\right)\right]-\alpha_{1}\right\}
$$

or

$$
u_{1} \simeq U_{1}\left(1+\left(n_{0} / n_{1}\right)\left\{\left[x_{1} / 2 M\left(r+n_{0} h\right) \alpha_{1}\right]-1\right\}\right) .
$$

If the high-pressure index of refraction is expressed (as suggested below) by

$$
n_{1}=n_{0}+k u_{1},
$$

the particle velocity using Eqs. (9) and (10) is obtained from

$$
u_{1} \cong \frac{\left(U_{1} k-n_{0}\right)+\left(\left(U_{1} k-n_{0}\right)^{2}+2 k n_{0} U_{1} x_{1}\left[M\left(r+n_{0} h\right) \alpha_{1}\right]^{-1}\right\}^{\frac{1}{2}}}{2 k} .
$$

After a shock front has interacted with the Mylar foil, the grid image displacement will not, in general, remain constant even if the shock pressure remains steady. This is because the optical path length through the shocked fluid of the light reflected from the foil increases with time as the shock front propagates toward the plate glass. Taking into account the refraction of the light beam through both a finite thickness of shocked liquid and the plate glass (using small angle approximations and assuming $U_{1}$ and $n_{1}$ are effectively constant), Eq. (10) may be rewritten in a more general form as

$$
u_{1} \cong \frac{U_{1}\left(x_{1} /\left(2 M \alpha_{1}\right)-\left[n_{0}\left(h+r_{g} / n_{g}\right)+\left(r_{1}-r_{g}\right)\right]\left(1-n_{1} / n_{0}\right)+r_{1}\left(n_{0} / n_{1}-1\right)\right\}}{\left(n_{1} / n_{0}\right)\left[n_{0}\left(h+r_{0} / n_{g}\right)+\left(r_{1}-r_{g}\right)\right]+r_{1}},
$$

where $r_{1}$ and $\left(r_{1}-r_{n}\right)$ are now the time-varying optical path lengths through the shocked and unshocked liquid. The thickness and refractive index of the glass are $r_{g}$ and $n_{g}$, respectively.

When two shock fronts are transmitted into the liquid (as illustrated by Fig. 3), the angle at which the second shock intersects the foil is obtained from

$$
\alpha_{2}=\alpha_{1}-\beta_{1} \text {. }
$$

Shock and particle velocities of the second shock front are given, respectively, by

and

$$
U_{2}=u_{1}+W \sin \alpha_{2} / M \tan \gamma_{12}
$$

$$
u_{2}=U_{2}-\left[\left(U_{2}-u_{1}\right) \tan \left(\alpha_{1}-\beta_{2}\right) / \tan \alpha_{2}\right] .
$$

Here $\beta_{2}$ is the foil turning angle as measured from its initial orientation. For small values of $\alpha$, if we neglect the optical path length through the shocked liquid with respect to the path length through the undisturbed liquid, the turning angle of the foil after interaction with the second shock is given by

$$
\beta_{2}=\alpha_{1}+\left(n_{0} / n_{2}\right)\left\{\left[x_{2} / 2 M\left(r+n_{0} h\right)\right]-\alpha_{1}\right\} .
$$

For small angles the second shock particle velocity may be obtained from Eqs. (14)-(17).

The shock and particle velocity of the single shock (film angle grid displacement is $\gamma_{02}$ in Fig. 4), which results from an initial shock being overtaken by the second shock, is calculated using Eqs. (1)-(13). Substitution of $\gamma_{20}$ for $\gamma_{01}$ and $x_{02}$ for $x_{1}$ in the equations is required.

\section{REFRACTIVE INDEX UNDER SHOCK}

Liquids in which the index of refraction under shock is to be measured are placed in immersed foil cells; these are mounted on 2024 aluminum or 356 brass driver plates. Plane shock waves are driven into the illuminated assemblies (Fig. 5) which are viewed with a streak camera. The quantities measured are: (a) the free-surface velocity of the aluminum or brass driver plate; (b) the velocity of the shock wave which is transmitted into the liquid; (c) the deflection of the grid light source image resulting from the interaction of the immersed foil with the shock.

To achieve a degree of redundancy in the index of refraction results, two foil assemblies are usually employed. These have foils at different angles (generally $4^{\circ}$ and $8^{\circ}$ ) to the driver plate. Explosive assemblies of this type are usually viewed by the streak camera via an optical path that includes a large, expendable mirror. This arrangement is used so that the explosive does

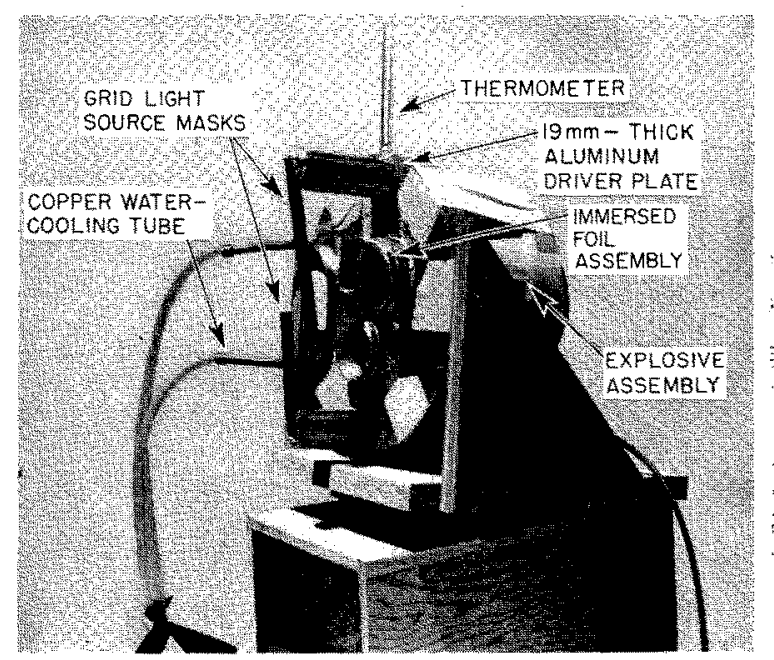

FIG. 5. Standard shot assembly for high-pressure index of refraction and Hugoniot data. 
not project the driver plate directly at the streak camera viewing port. Because the data analyses are simplified by adhering closely to the idealized geometry of the optical paths shown in Fig. 1, a special effort is made to view both the $4^{\circ}$ and $8^{\circ}$ at foil very nearly normal incidence. To accomplish this, the shot assembly is placed so that the $4^{\circ}$ foil assembly is perpendicular to the optical path to the streak camera objective lens (via the expendable mirror). In addition, a small auxiliary mirror inclined at an angle of one-half the difference between the two foil-assembly angles (or $2^{\circ}$ in the present example) is cemented onto the large mirror. The experiment is aligned so that the streak camera observes the $8^{\circ}$ foil assembly via the auxiliary inclined mirror and the remainder of the experiment via the large mirror; thus, both foil assemblies appear normal to the streak camera (Fig. 6). The alignment of the actual grid with its image on the Mylar foil as seen by the camera provides pictorial verification that the desired geometrical relations have been achieved.

In order to closely describe the initial and shock states achieved within the liquid, the initial temperature of the driver plate -and therefore essentially that of a liquid which contacts it over a 3 -cm-diam circle-is kept from rising appreciably above ambient temperatures. This is accomplished by flowing water through the copper tubing as shown in Fig. 5. The procedure is important in warm weather and also because of the heating of the assemblies that occurs as a result of exposure to intense light from photographic lamps during optical alignment procedures.

The Hugoniot states in the buffer fluids are obtained using the impedance-match method. ${ }^{2}$ The free-surface velocity of the aluminum or brass driver plates (obtained from the light cutoff angle of the inclined mirror mounted on the driver plate, Figs. 5 and 6 ) is used to obtain the Hugoniot state in the driver plate required for the impedance-match solution. The shock velocity in each of the two liquid cells in the assembly is determined separately, using Eq. (3). The shock velocities obtained from each foil assembly differ typically by $1.5 \%$; these numbers have been averaged for inclusion in Table I. The uncertainties arise mostly from un-

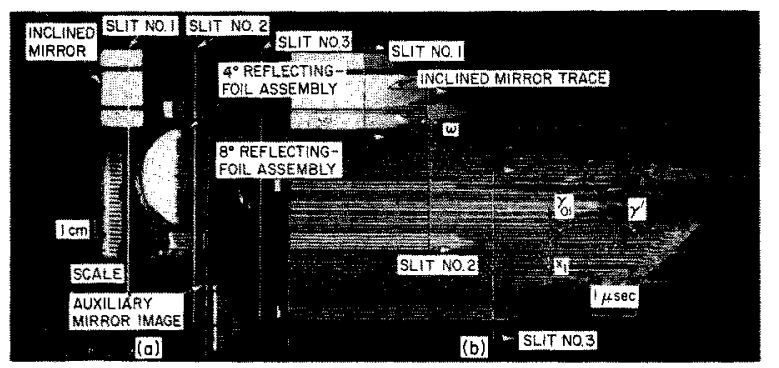

Fig. 6. Streak camera record, shot 10639 . Standard shot for index of refraction and Hugoniot data. (a) View assembly as seen by streak camera (still) via expendable and auxiliary mirrors. (b) Resulting streak record showing angle $\gamma_{01}$ of grid image displacement $x_{1}$, angle of shock tilt $\omega$, and angle of foil disturbance $\gamma^{\prime}$.

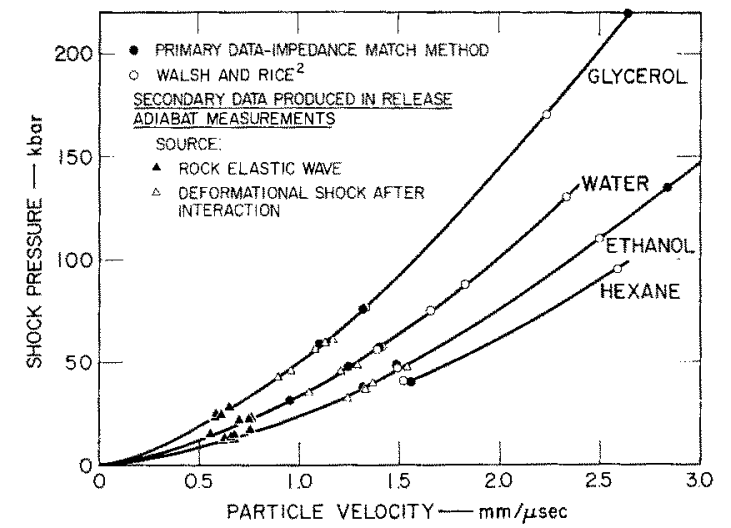

FIG. 7. Hugoniot data for glycerol, water, ethanol, and hexane.

certainties of the foil cutoff angle and the optical magnification.

The Hugoniot data indicated as primary data in Fig. 7 are in good agreement with those reported by Walsh and Rice. ${ }^{2}$ Also shown in Fig. 7 are Hugoniot points, centered at zero pressure, obtained in the course of performing release adiabat measurements on polycrystalline quartz rock. ${ }^{6}$ Although three different shock states are recorded in these experiments (Fig. 3), only two of these - the shock state induced in the liquid by the elastic wave in the rock and that resulting from the second shock in the liquid overtaking the first shock in the liquid - represent states which are achieved from ambient pressure by a single shock front; these states lie on the Hugoniot of the liquid which is centered at zero pressure. Although these states are plotted for reference in Fig. 7, they should be considered secondary data for liquids, since they depend indirectly on the accuracy of the impedance-match "standard" experiments for the high-pressure index of refraction. These Hugoniot states are also more scattered than those of the "standard shots," presumably because of the fluctuations in time and space of shocks transmitted from the rocks into the liquids.

The index of refraction under shock is calculated using the shock velocity, the grid-image displacements, and the geometrical parameters of the shot assembly. The particle velocity is assumed to be known from the impedance-match solution. The index of refraction of the liquids is obtained by solving $\mathrm{Eq}$. (10) for $n_{1}$. The refractive index obtained from two foil assemblies on the same shot generally agreed within $1 \%$ when computed from the average of the shock velocities. This uncertainty, is comparable to the combined uncertainties of the grid-image displacements measured on the film. The maximum uncertainty in a refractive index determination from each foil assembly is typically $\pm 1.3 \%$. This error arises largely from a maximum uncertainty of $2.5 \%$ in liquid particle velocity due to the uncertainty in the driver plate free-surface velocity. The values for the refractive index, average values in the case of two cells, are given in Table I and plotted in Fig. 8. 
TABLE I. Index of refraction vs shock stress and density for various liquids.

\begin{tabular}{|c|c|c|c|c|c|c|c|c|c|}
\hline Liquid & Shot No. & $\begin{array}{c}\text { Driver } \\
\text { free- } \\
\text { surface } \\
\text { velocity } \\
(\mathrm{mm} / \mu \mathrm{sec})\end{array}$ & $\begin{array}{c}\text { Initial } \\
\text { tem- } \\
\text { perature } \\
\left({ }^{\circ} \mathrm{C}\right)\end{array}$ & $\begin{array}{c}\text { Shock } \\
\text { velocity } \\
(\mathrm{mm} / \mu \mathrm{sec})\end{array}$ & $\begin{array}{c}\text { Particle } \\
\text { velocity } \\
(\mathrm{mm} / \mu \mathrm{sec})\end{array}$ & $\begin{array}{l}\text { Shock } \\
\text { stress } \\
\text { (kbar) }\end{array}$ & $\begin{array}{l}\text { Density } \\
\left(\mathrm{g} / \mathrm{cm}^{3}\right)\end{array}$ & $\begin{array}{l}\text { Index of } \\
\text { refraction } \\
\text { (measured) }\end{array}$ & $\begin{array}{l}\text { Index of } \\
\text { refraction } \\
\text { (Lorentz- } \\
\text { Lorenz) }\end{array}$ \\
\hline \multirow{5}{*}{ Glycerola,b } & $\ldots$ & $\ldots$ & $\ldots$ & $\ldots$ & 0 & 0 & 1.249 & $1.468^{\circ}$ & $1.468^{\mathrm{c}}$ \\
\hline & $10641^{d}$ & 1.40 & 28 & 4.28 & 1.10 & 59.0 & 1.681 & $1.616^{\mathrm{c}}$ & $1.679^{\circ}$ \\
\hline & $10279^{\circ}$ & 1.77 & 25 & 4.54 & 1.32 & 75.0 & 1.764 & $1.667^{\circ}$ & $1.723^{\circ}$ \\
\hline & $10389^{\mathrm{e}}$ & 1.77 & 25 & 4.59 & 1.32 & 75.6 & 1.754 & $1.656^{\circ}$ & $1.720^{\mathrm{e}}$ \\
\hline & $11884^{\mathrm{f}}$ & 3.23 & 25 & 6.67 & 2.64 & 220.0 & 2.08 & 1.755 & 1.885 \\
\hline \multirow[t]{3}{*}{ Water ${ }^{b \cdot g}$} & . & $\ldots$ & $\cdots$ & $\cdots$ & 0 & 0 & 0.9982 & 1.333 & 1.333 \\
\hline & $10640^{\mathrm{d}}$ & 1.55 & 28 & 3.87 & 1.245 & 48.0 & 1.468 & 1.474 & 1.517 \\
\hline & $10383^{\circ}$ & 1.78 & 28 & 4.09 & 1.40 & 58.0 & 1.515 & 1.482 & 1.537 \\
\hline \multirow[t]{4}{*}{ Ethanol ${ }^{\mathrm{b} \cdot \mathrm{g}}$} & $\ldots$ & $\cdots$ & & $\cdots$ & 0 & 0 & 0.7894 & 1.362 & 1.362 \\
\hline & $10639 d$ & 1.55 & 30 & 3.74 & 1.32 & 38.0 & 1.200 & 1.564 & 1.601 \\
\hline & $10700^{\circ}$ & 1.77 & 28 & 4.13 & 1.485 & 48.0 & 1.225 & 1.562 & 1.609 \\
\hline & $11884^{f}$ & $3.23^{f}$ & 25 & 6.04 & 2.84 & 135.0 & 1.490 & 1.688 & 1.777 \\
\hline \multirow[t]{3}{*}{ Hexane } & $\ldots$ & $\ldots$ & $\cdots$ & $\ldots$ & 0 & 0 & 0.6603 & 1.375 & 1.375 \\
\hline & $10279^{e}$ & 1.77 & 25 & 4.17 & 1.52 & 41,3 & 1.039 & 1.643 & 1.638 \\
\hline & $10431^{\circ}$ & 1.82 & 26 & 3.92 & 1.56 & 40.5 & 1.096 & 1.684 & 1.689 \\
\hline
\end{tabular}

a $96.5 \% \mathrm{C}_{2} \mathrm{H}_{8} \mathrm{O}_{3} ; 3.5 \% \mathrm{H}_{2} \mathrm{O}$ by weight.

b Initial densities: glycerol, $1.2492 \pm 0.0005 \mathrm{~g} / \mathrm{cm}^{3}$ (measured at $23.8^{\circ} \mathrm{C}$ ); water, $0.9982 \mathrm{~g} / \mathrm{cm}^{8}$ (handbook at $20^{\circ} \mathrm{C}$ ); ethanol, $0.7894 \mathrm{~g} / \mathrm{cm}^{3}($ handbook at $20^{\circ} \mathrm{C}$ ); hexane, $0.6603 \mathrm{~g} / \mathrm{cm}^{3}$ (handbook at $20^{\circ} \mathrm{C}$ ).

- Initial refractive index calculated from $n=0.0965 n_{g}+0.0035 n_{w}$, where $n_{0}$ and $n_{w}$ are refractive indices of pure gylcerol and water.

d Explosive system: P-80 plane-wave generator, $51 \mathrm{~mm}$ Barato1, $17 \mathrm{~mm} 2024$ aluminum.

explosive system: P-80 plame-wave generator, $13 \mathrm{~mm}$ composition B-3,51 mm Baratol, $17 \mathrm{~mm} 2024$ aluminum.

tExplosive system: P-80 plane-wave generator, $102 \mathrm{~mm} 9404$ (HMX), $1.6 \mathrm{~mm}$ air, $6.4 \mathrm{~mm} 346$ brass (flyer $\mathrm{ylate}$ ), $38 \mathrm{~mm}$ air (free run), $3.2 \mathrm{~mm} 346$ brass.

8 Initial density value corrected for temperature in calculations.

Although the available index of refraction data can be represented by first- or second-order polynomials in the index of refraction-density plane, for computational purposes it is also convenient to express the refractive index in terms of shock particle velocity (Table II) as in $\mathrm{Eq}$. (11). The linear relation between liquid particle velocity and refractive index, as listed in Table II, neglects presently unknown differences in the refractive index of a liquid that achieves the same particle velocity via different paths, e.g., one or more shock transitions.

TABLE II. Relation of index of refraction to shock particle velocity and to density.

\begin{tabular}{|c|c|c|c|c|c|}
\hline \multirow[t]{2}{*}{ Liquid } & \multicolumn{2}{|c|}{$n_{1}=n_{0}+k u$} & \multicolumn{3}{|c|}{$n_{1}=a+b \rho+c \rho^{2}$} \\
\hline & $n_{0}$ & $\begin{array}{c}k \\
(\mu \mathrm{sec} / \mathrm{mm})\end{array}$ & $a$ & $\begin{array}{c}b \\
\left(\mathrm{~cm}^{3} / \mathrm{g}\right)\end{array}$ & $\underset{\left(\mathrm{cm}^{6} / \mathrm{g}^{2}\right)}{c}$ \\
\hline \multirow[t]{2}{*}{ Glycerol } & 1.468 & 0.122 & 1.0180 & 0.3594 & s \\
\hline & & & 0.8674 & 0.5587 & -0.06305 \\
\hline Water & 1.333 & 0.111 & 1.0340 & 0.2937 & a \\
\hline \multirow[t]{2}{*}{ Ethanol } & 1.362 & 0.124 & 0.9916 & 0.4692 & a \\
\hline & & & 0.9504 & 0.5513 & -0.03796 \\
\hline Hexane & 1.375 & 0.188 & 0.9071 & 0.7085 & s \\
\hline
\end{tabular}

s Only first-order polynomial fitted.

The variation with temperature and pressure of the index of refraction in a variety of optically transparent media has been fit to various formulas. Although many of these are empirical, several are based in form on, or are corrections to, the Lorentz-Lorenz formula, which gives the refractive index of a dielectric material $n$, in terms of density $\rho$, polarizability $P$, molecular weight $M$, and Avogadro's number $N_{0}$, as

$$
\left(n^{2}-1\right) /\left(n^{2}+2\right) \rho=4 \pi N_{0} P / 3 M=L .
$$

The fact that the quantity $L$ has been observed to remain nearly constant in certain media over a wide range of temperature and pressure has been employed in the use of the index of refraction to determine density changes in static high-pressure measurements, such as those of Smith and Pings ${ }^{9}$ in solid argon and in the weak shock experiments of Flook and Homig ${ }^{10}$ in a variety of fluids. To compare the predictions of the LorentzLorenz formula for the index of refraction to that observed under shock, Eq. (18) is recast in terms of the compression $\left(\rho / \rho_{0}\right)$, with the assumption that $L$ is constant. This gives

$$
n_{1}=\left[\frac{n_{0}^{2}+2+2\left(n_{0}^{2}-1\right)\left(\rho / \rho_{0}\right)}{n_{0}^{2}+2-\left(n_{0}^{2}-1\right)\left(\rho / \rho_{0}\right)}\right]^{\frac{1}{2}} .
$$

The initial liquid density is $\rho_{0}$.

The disagreement between the observed refractive index values and those calculated from the LorentzLorenz formula (Eq. 19) is believed to be experimentally significant. The present index of refraction data for water are in good agreement with the results of $\mathrm{Zel}^{\prime}$ dovich et $a ._{.11}$; these are the only other quantitative optical data reported for liquids at these pressure levels. In that work, both a geometric and a photometric tech-

${ }^{9}$ B. L. Smith and C. J. Pings, J. Chem. Phys. 38, 825 (1963). ${ }^{10}$ W. M. Flook, Jr., and D. F. Hornig, J. Chem. Phys. 23, 816 (1955).

11 Y. B. Zel'dovich, S. B. Kormer, M. V. Sinitsyn, and K. B. Yushko, Soviet Phys.-Doklady 6, 494 (1961). 


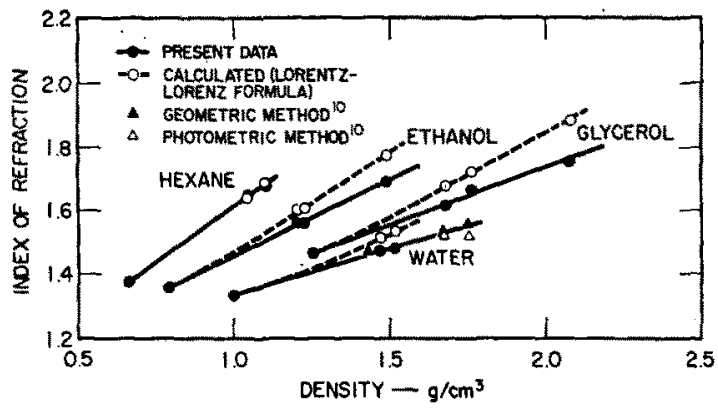

FIG. 8. Index of refraction versus density for glycerol, water, ethanol, and hexane.

nique were used to observe the index of refraction of water up to $\sim 150 \mathrm{kbar}$ (Fig. 8). Zel'dovich et al. suggest that the geometric method, which is analogous to the present method, is more accurate. The results from the geometric technique agree more closely with the present results than do the photometric data.

The increase reported here in index of refraction of water with density exceeds that measured by Erkman and Christensen..$^{12}$ The difference is thought to arise from uncertainties in determination of the shock state in water, to which the index of refraction measurement is quite sensitive.

\section{OTHER APPLICATIONS OF THE IMMERSED-FOIL METHOD}

\section{Adiabatic Sound Velocity of a Liquid in the Shocked State}

In the course of analyzing streak camera records from the high-pressure index of refraction experiments, a small pulse-like deflection of the grid images was observed after arrival of the shock front at the foil (Fig. 6). This disturbance travels along the foil, ${ }^{13}$ which has already been turned through an angle $\beta_{1}$. with apparent velocity

$$
U_{a}^{\prime}=W \cos \alpha / M \cos \beta_{1} \tan \gamma^{\prime} .
$$

Here $\gamma^{\prime}$ is the angle of the disturbance on the streak camera film. It was noted that the apparent velocity $U_{a}^{\prime}$ is far less than the apparent velocity of the initial shock, $U_{1}{ }^{\prime}$. Although the pulse-like disturbance is discemible on the streak records of nearly all the index of refraction experiments, it appears more prominently on the records from the water, hexane, and ethanol shots than on those of the glycerol shots. This, and the relation in time of the pulse disturbance to the shock arrival, suggests that the disturbance is produced as a result of the shock impedance mismatch of

${ }^{12}$ J. O. Erkman, A. B. Christensen, and G. R. Fowles, Stanford Research Institute Project 5386, Contract AF 29(601)-6427, Final Report (1965).

${ }^{13}$ In the direction parallel to the undisturbed foil, i.e., perpendicular to the direction to the streak camera. the Mylar foil with the liquid ${ }^{14}$ in the following manner. Upon interaction of a single shock in the liquid with the Mylar foil, a small disturbance is generated as the Mylar achieves the same pressure and particle velocity as the liquid; this disturbance, as indicated in Fig. 9, is reflected back into the shocked liquid. The disturbance will propagate at a velocity $c$, as measured in the coordinate system which is fixed with respect to the shocked liquid. The wavefront associated with this rearward-traveling disturbance lies at an angle

$$
\varphi=\alpha+\tan ^{-1}\left[c \sin \alpha_{1} \cos \alpha_{1} /\left(U+c \sin ^{2} \alpha_{1}\right)\right],
$$

with respect to the plane of the undisturbed liquiddriver plate interface.$^{15}$ Because of the shock tilt which is nearly always present in plane shock wave experiments, the liquid-driver interface has been turned through an angle

$$
\psi=\tan ^{-1}\left[u \cos \Omega \sin \Omega /\left(U-u \sin ^{2} \Omega\right)\right]
$$

by the shock. Upon arriving at the liquid-driver plate interface the disturbance is reflected at an angle $\varphi-\psi$ with respect to the normal to the turned interface. The sign convention for $\Omega$, and hence that for $\psi$, both follow from the definition of $\omega$ as used in Eq. (4). Upon reflection at the liquid-driver interface, the disturbance is incident at the angle $\alpha-\beta_{1}-2 \psi$ on the now turned Mylar foil. The arrival of this disturbance at the foil produces the small deflection of the grid light source image that is observed to travel along the foil with apparent velocity $U_{a}^{\prime}$. In the case of an approximately steady shock, the propagation velocity, $c$ of the foil disturbance in the liquid should be essentially that of the adiabatic sound velocity of the liquid in the highpressure shock state. The velocity, from Eqs. (20)-(22)

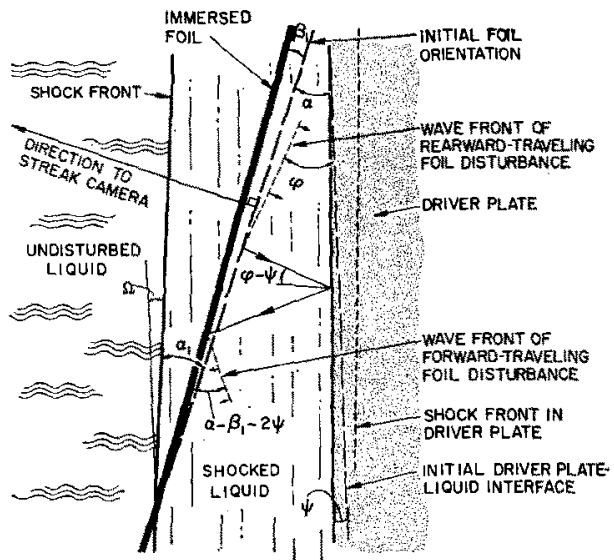

Fic. 9. Relation of path of foil disturbance (reverberating between Mylar foil and driver plate through liquid in shock state), foil angle, and shock tilt.

\footnotetext{
14 Mylar apparently matches the shock impedance of glycerol more closely than the other liquids.

${ }^{16}$ For simplicity the subscripts " 1 " on $u, U$, and $\Omega$, indicating the first shock front, are not used.
} 
and Fig. 9, is given by

$c=U_{a}^{\prime}\left[\sin \left(\alpha-\psi-\beta_{1}\right)+\cos \left(\alpha-\psi-\beta_{1}\right) \tan (\varphi-\psi)\right]$.

In the present index of refraction experiments the shock pressure in the liquid between the Mylar foil and driver plate must decay somewhat during the time the foil disturbance propagates from the foil to the liquid-driver interface and back again to the foil. The pressure decay is due to the characteristic shock profile induced by in-contact detonation of a finite thickness explosive pad (rather than from rarefactions arriving from the rear surface of the driver plate). ${ }^{16}$ Hence, because of the variation in shock pressure, the application of Eqs. (21)-(23) using the present records serves primarily an illustrative purpose, al though the numerical results are probably only slightly in error. Applying Eqs. (21)-(23) to (for example) the ethanol data at $38 \mathrm{kbar}$ in shot 10,639 (Fig. 6), gives for $c, 5.3$ and 5.7 $\mathrm{mm} / \mu \mathrm{sec}$. These values were calculated from the $8^{\circ}$ and $4^{\circ}$ foil assemblies, respectively. The values of the adiabatic sound velocity for states at some pressure $P$ on the Hugoniot curve should in general be somewhat less than the Hugoniot sound velocity or

$$
c \leq c^{\prime}
$$

where the Hugoniot sound velocity is defined as

$$
c^{\prime}=(d P / d \rho)^{\frac{1}{2}} \mid \text { Hug. }
$$

The value of $c^{\prime}$ obtained at $38 \mathrm{kbar}$ for ethanol was 6.1 $\mathrm{mm} / \mu \mathrm{sec}$, which satisfies the inequality given by $\mathrm{Eq}$. (24). These results, although semiquantitative because of pressure attenuation, indicate that the immersed foil method should be applicable to the measurement of the adiabatic sound velocity of a liquid in the highpressure shocked state. It should be emphasized that measurement of the adiabatic sound velocity using the immersed-foil method should be valid in experiments in which a truly steady shock wave is induced in the liquid. This may be achieved in practice by impacting the assembly with a flyer plate of sufficient thickness that the foil disturbance may propagate back and forth between the foil and the driver plate within liquid at a steady pressure before the arrival of rarefactions from the rear of the flyer plate.

\section{Shock Attenuation Studies}

The immersed-foil method is being applied to the study of shock wave attenuation in solids. ${ }^{12}$ Experi-

\footnotetext{
${ }^{16}$ B. G. Craig, in Tenth International Symposium on Combustion, Cambridge, England (The Combustion Institute, Pittsburgh, 1965).
}

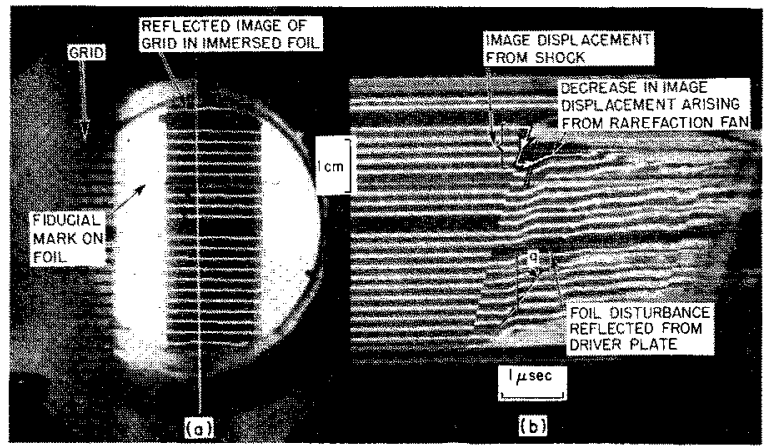

Fig. 10. Streak camera record, shot 11 190, showing shock profle transmitted into water by flyer impact. Driver plate impacted by 2 -mm-thick aluminum flyer plate traveling at 1.3 $\mathrm{mm} / \mu \mathrm{sec}$. (a) View of foil assembly as seen by streak camera (still). (b) Streak record showing increase and decrease of image displacement produced by shock and rarefaction. Reflected foil disturbance at angle $q$ is also indicated.

ments are performed in which explosively accelerated flyer plates strike a target plate and the resulting shock disturbance is transmitted into an immersed-foil assembly. The flyer plate and target plate are usually constructed of the material in which shock attenuation is to be studied. The image of a grid light source as reflected in a Mylar foil is viewed, as in the present experiments, with a streak camera. Streak camera records such as that shown in Fig. 10. have been used for measuring the velocity of the initial unloading wave of the rarefaction fan which originates from the rear (free) surface of the flyer plate. The velocity, as well as the pressure decrease associated with the initial unloading wave, are important data sought in shock attenuation experiments.

\section{ACKNOWLEDGMENTS}

This research was supported in part through the Defense Atomic Support Agency, Contract DA-49-146$\mathrm{XZ}-277$, as a portion of the Advanced Research Projects Agency's VELA-UNIFORM program. We appreciate the assistance of our colleagues, A. B. Christensen, B. Y. Loo, and J. T. Rosenberg in deriving formulas and carrying out experimental analyses, and that of W. Isbell in the careful execution of several experiments. We thank J. O. Erkman for providing us with Fig. 10. Discussions with W. Isbell and J. K. Crosby concerning the optical systems were also extremely helpful. We also wish to thank Dr. G. R. Fowles for his numerous suggestions and guidance and Dr. D. G. Doran for reviewing the manuscript. 